\title{
Behavioral, Neuroplasticity and Metabolic Effects of 7,8-dihydroxy-4-methylcoumarin Associated With Physical Activity in Mice
}

\section{Priscilla Karla Fernandes Lopes}

Federal University of Lavras: Universidade Federal de Lavras

Daiane Engel ( $\nabla$ daiane.engel01@gmail.com)

unicamp https://orcid.org/0000-0002-1950-196X

\section{Moises Silvestre de Azevedo Martins}

Federal University of Lavras: Universidade Federal de Lavras

\section{Natalia Oliveira Bertolini}

Federal University of Lavras: Universidade Federal de Lavras

\section{Chrystian Araujo Pereira}

Federal University of Lavras: Universidade Federal de Lavras

\section{Licio Augusto Velloso}

UNICAMP: Universidade Estadual de Campinas

\section{Sergio Scherer Thomasi}

Federal University of Lavras: Universidade Federal de Lavras

\section{Rodrigo Ferreira de Moura}

Federal University of Lavras: Universidade Federal de Lavras

\section{Research Article}

Keywords: Coumarin, DHMC, VEGF, fasting glucose, anxiolytic, physical activity

Posted Date: May 21st, 2021

DOI: https://doi.org/10.21203/rs.3.rs-484789/v1

License: (1) (1) This work is licensed under a Creative Commons Attribution 4.0 International License. Read Full License 


\section{Abstract}

The search for strategies to develop resilience against metabolic and neuropsychiatric disorders has motivated the clinical and experimental assessment of early life interventions such as lifestyle-based and use of unconventional pharmacological compounds. In this study, we assessed the effects of voluntary physical activity and 7,8-Dihydroxy-4-methylcoumarin (DHMC), independently or in combination, over mice physiological and behavioral parameters, adult hippocampal and hypothalamic neurogenesis, and neurotrophic factors expression in the hypothalamus. C57BI/6J mice were submitted to a 29-day treatment with DHMC and allowed free access to a running wheel. We found that DHMC treatment reduced fasting blood glucose levels. Moreover, physical activity showed anxiolytic effect in the elevated plus maze task and DHMC produced additional anxiolytic behavior, evidenced by reduced activity during the light cycle in the physical activity group. Although we did not find any differences in hypothalamic or hippocampal adult neurogenesis, DHMC increased gene expression of VEGF, which was correlated to the reduced fasting glucose levels. In conclusion, our data emphasize the potential of physical activity in reducing development of neuropsychiatric conditions, such as anxiety, and highlights DHMC as an attractive compound to be investigated in future studies addressing neuropsychiatric disorders associated with metabolic conditions.

\section{Introduction}

Disruption of synaptic plasticity processes, neuronal growth and remodeling, neurogenesis, and eventually, progressive neuronal loss, underlies the development of several neuropsychiatric and metabolic disorders (Myers and Olson 2012; Dugger and Dickson 2017; Estrada and Contreras 2019). As an attempt to counteract such outcomes, early life interventions, mainly lifestyle-based and new phytochemical compounds, have been investigated aiming at preventing neurodegeneration and promoting healthier aging (Jameel et al. 2016; Alkadhi 2018; Carrera et al. 2020).

Coumarins are polyphenols that constitute a large class of heterocyclic oxygen compounds, initially found as secondary plant metabolites. They have high bioavailability, low molecular weight and simple processes for synthesis (Wu et al. 2009). Coumarins have demonstrated experimental therapeutic potential in several diseases, including obesity, diabetes, cardiovascular failure, renal failure, cancer, and neurological disorders (Kadakol et al. 2016; Ahmed et al. 2020). Notably it was reported that they increased insulin secretion in isolated cells from mouse pancreas (Ahmed et al. 2020) and prevent oxidative damage in myocardial infarction in rats (Rajadurai and Stanely Mainzen Prince 2006). Regarding the central nervous system (CNS) these molecules have shown experimental results in neuroprotection in human (Molina-Jiménez et al. 2005), mouse (Yao et al. 2015) and rat (Kang and Kim 2007) neuronal cells in vitro, adult neurogenesis, cognitive function improvement in mice (Gao et al. 2015) and antidepressant effect in rats (Yang et al. 2020). The 7,8-Dihydroxy-4-methylcoumarin (DHMC) is a coumarin synthetized by relatively simple, low-cost and good yielding processes (Potdar et al. 2001). It is considered a simple coumarin since structural substitution occurs only in the benzene ring using two hydroxyl radicals. In vitro studies have pointed to a possible neuroprotective effect of this coumarin, 
mainly related to its antioxidant and anti-inflammatory properties (Tyagi et al. 2005; Togna et al. 2014; Jin et al. 2015). In the CNS coumarins seem to improve cell survival, promoting neuroprotection and promoting favorable conditions for neuronal expansion in mice and rats (Gao et al. 2014; SkalickaWoźniak et al. 2016; Qin et al. 2017).

Physical activity is widely known to reduce the risk of metabolic abnormalities, such as obesity (Shapiro et al. 2011; Swift et al. 2018), as well as to prevent or slow down the progression of neuropsychiatric conditions, such as dementia, depression and anxiety (Angevaren et al. 2008; Cunha et al. 2013; Kandola et al. 2018). Among mechanisms that have been shown to mediate physical activity effects in the CNS are the increased expression of neurotrophic factors and synaptic plasticity markers, decrease in neuroinflammatory processes (Cotman et al. 2007; Choi et al. 2018) and enhancement of adult neurogenesis (van Praag et al. 1999; Kronenberg et al. 2006; Niwa et al. 2016). The proliferation and differentiation of adult born neurons can be stimulated by physiological and external stimuli, such as physical activity and antidepressants (van Praag et al. 1999; Santarelli et al. 2003; Niwa et al. 2016). Frequently these stimuli-induced neuroplasticity responses are mediated by trophic factors, such as ciliary neurotrophic factor (CNTF), brain-derived neurotrophic factor (BDNF) and vascular endothelial growth factor (VEGF) (Fabel et al. 2003; Kokoeva et al. 2007; Choi et al. 2018).

There is a growing interest in the search for strategies to efficiently improve resilience against the development of metabolic and neuropsychiatric diseases. In the present study we evaluated the impact of a pharmacological and a behavioral intervention (coumarin treatment and physical activity, respectively) in promoting metabolic fitness and brain plasticity in normal mice. We show that, either isolated or combined, coumarin and physical activity produce behavioral improvements and neuroplasticity responses, particularly in neurotrophic factors expression in the hypothalamus. These results expand the window of opportunities for the development of preventive and therapeutic approaches in metabolic and neuropsychiatric conditions.

\section{Methods}

\section{Chemicals}

The coumarin 7,8-Dihydroxy-4-methylcoumarin (DHMC) was synthesized at the Chemistry Department of the Federal University of Lavras (UFLA) as previously described (Potdar et al. 2001). The synthesis process resulted in $97 \%$ purity and approximately $75 \%$ yield DHMC.

\section{Experimental animals}

Adult male C57BL/ 6 mice 8 weeks old, obtained from Federal University of Minas Gerais (UFMG), were kept in cages of one at as temperature of $22 \pm 2{ }^{\circ} \mathrm{C}$, under a $12 \mathrm{~h}$ light/dark cycle and with free access to food and water. The procedures were approved and carried out in accordance with the Ethics Committee of the Federal University of Lavras/Brazil and Animal Experiments Control Council (CONCEA), according 
to protocol $n^{\circ} 078 / 17$. All procedures were focused on reducing the number of animals used and minimizing their suffering.

\section{Experimental protocol}

For the administration of coumarin and voluntary physical activity wheel, the mice were divided into four groups ( $\mathrm{n}=8 /$ group): vehicle, coumarin, physical activity + vehicle, physical activity + coumarin (30 mg / weight / day). During the habituation period ( 2 weeks), a $Z$ distribution of the individuals in the groups was performed according to the running volume for 72 hours before the beginning of the experiment. "The animals were distributed in the four groups, following $Z$ distribution of the physical activity volume acquired 72 hours before the beginning of the experiment (from higher volume to lower volume of physical activity)". For the other experiments, the animals were randomly divided into smaller groups, meeting some technical limitations. Being distributed: elevated plus maze experiment, five $(n=5)$ animals from each group were used. In the molecular tests, the eight initial animals were divided into two groups of four animals $(n=4)$, distributed between immunofluorescence assays and detection of neurotrophic markers by polymerase chain reaction (PCR). Coumarin was administered by gavage once a day, for 29 days (dissolved in saline solution containing $0.3 \%$ sodium carboxymethylcellulose, $\mathrm{CMC}-\mathrm{Na}$ ). Control groups received an equal volume of $0.3 \%$ CMC-Na saline (vehicle) (Fig. 1A). BrdU (5-bromo-2'deoxyuridine; Sigma) was used in the first 10 days to assess cell proliferation and neurogenesis. BrdU is an analogue of thymidine that is incorporated into the DNA double helix during the $S$ phase of the cell cycle and therefore marks cells in active proliferation 23. All animals received $\mathrm{BrdU}(0.1 \mathrm{M}$, phosphate buffered saline [PBS], $\mathrm{pH}=7.2 ; 50 \mathrm{mg} /$ weight, $2 \mathrm{x} /$ day ip, 12/12 hours) and were sacrificed 20 days later to test cell proliferation and early neuronal differentiation,

To promote physical activity through voluntary exercises, two groups (physical activity and physical activity + coumarin) had free access to the racing wheel for 29 days. The casters were installed inside the cage and have a speed sensor connected to an interface and program to record this information (Columbus Instruments ${ }^{\circledR}$ ). At the end of the experiment, the total volume of voluntary physical activity in the period and the average speed were quantified and stratified in the light and dark periods to assess behavioral paradigms.

\section{Behavioral analysis}

For analysis of anxious behavior and locomotion, we use the Elevated Plus Maze (EPM). EPM is made of polished wood panels with a matte dark acrylic surface and consists of two open arms $(50 \times 10 \times 0 \mathrm{~cm})$ and two closed arms $(50 \times 10 \times 40 \mathrm{~cm})$ that extended from a common central platform $(5 \times 5 \mathrm{~cm})$ elevated $50.0 \mathrm{~cm}$ from the floor. Mice were individually placed on the central platform facing an open arm and allowed to freely explore the maze for $5 \mathrm{~min}$. Behavior in the EPM trials was scored using video recordings as follows: entries into open arms (all four limbs crossing into an open arm), time in open arms (duration of time spent in open arms), entries into closed arms (all four limbs crossing into a closed arm), time in closed arms (duration of time spent in closed arms). The data were considered in relation to the total number of entries. Illumination was reduced by means of high lights with equal light intensity 
between a periphery and a central part, as well as between open and closed arms. At the end of each behavioral session, the devices were cleaned with a 70\% alcohol solution and an interval of 5 minutes between the tests.

\section{Immunofluorescence staining}

On the 30th day of the experiment, mice were anesthetized with a mixture of ketamine ( $45 \mathrm{mg} / \mathrm{wt}, \mathrm{ip})$ and xylazine ( $5 \mathrm{mg} / \mathrm{wt}, \mathrm{ip})$ and perfused through the left cardiac ventricle with $0.9 \%$ saline solution, followed by $4 \%$ paraformaldehyde (PFA) in $0.1 \mathrm{M}$ PBS ( $\mathrm{pH} 7.4)$. After perfusion, the brains were removed, post-fixed in the same fixative solution for $24 \mathrm{~h}$ at room temperature (RT), and immersed in a $30 \%$ sucrose solution in PBS at $4^{\circ} \mathrm{C}$. Serial coronal sections $(20 \mu \mathrm{m})$ of were obtained with a cryostat (LEICA Microsystems, CM1860). To analyze cell proliferation and neurogenesis in the hippocampus and hypothalamus, a series of one-in-six free floating sections were processed for detection of the BrdU immunoreactivity.

The neural progenitor phenotype was assessed by double labeling BrdU and doublecortin (DCX, an early neuronal differentiation marker). Briefly, after DNA denaturation in $2 \mathrm{~N} \mathrm{HCl}$ at room temperature (RT) for 1 h and pre-incubation with $10 \%$ blocking solution (0.1M PBS with $10 \%$ normal goat or donkey serum and $0.2 \%$ Triton X-100), sections were incubated overnight at $4{ }^{\circ} \mathrm{C}$ in anti-BrdU (rat, 1:200, AB6326; ABCAM) and anti-DCX (rabbit,1:100[PK1] ; 4604S; Cell Signaling) primary antibodies. The sections were then incubated with secondary antibodies anti-rat (donkey, 1:500; A-21208, Invitrogen) e anti-rabbit (donkey, 1:500, A10040; Invitrogen) for $2 \mathrm{~h}$ at RT.

The morphological analyses were performed on coded slides, with the executing researcher blinded to the experimental group. The total numbers of BrdU-immunopositive cells were estimated by manually counting all positive cells in coronal sections from 1.06 to $3.52 \mathrm{~mm}$ posterior to Bregma, to include the hypothalamic and hippocampal neurogenic niches (estimated as 120 sections).

The results were expressed as the total number of labeled cells by multiplying the total number of labeled cells obtained from the sum of every one-in-six section by the serial sectioning factor (obtained total cell counting $\times$ 6). Double-labeling was confirmed by three-dimensional reconstruction of z-series of confocal microscopy covering the entire nucleus (or cell) of interest (confocal microscope Upright LSM780-NLO).

\section{RNA extraction and quantitative real-time PCR}

RNA samples were prepared using TRIzol (Invitrogen) according to the manufacturer's recommendations. Spectrophotometry was employed for RNA quantification. For each sample, $2 \mu \mathrm{g}$ of RNA was employed for the synthesis of complementary DNA (cDNA) using the High Capacity cDNA Reverse Transcription Synthesis kit (Applied Biosystems) according to the manufacturer's recommendations. Real-time PCR reactions were run using the TaqMan system (Applied Biosystems). Primers used were DCX (Mm00438400_m1); NEUROD1 (Mm01946604_s1); CNTF (Mm00446373_m1), CNTFR (Mm00516693_m1), BDNF (Mm01334043_m1) and VEGF (Mm00437306_m1). Analyses were run using $4 \mu \mathrm{L}(10 \mathrm{ng} / \mu \mathrm{L}) \mathrm{cDNA}, 0.625 \mu \mathrm{L}$ primer/probe solution, $1.625 \mu \mathrm{L} \mathrm{H} 2 \mathrm{O}$, and $6.25 \mu \mathrm{L} 2 \mathrm{X}$ TaqMan Universal 
MasterMix. GAPDH (Mm99999915_g1) was employed as a reference gene. Gene expression was obtained using the SDS System 7500 software (Applied Biosystems).

\section{Blood glucose analysis}

For the fasting glucose test the animals were subjected to 8 hours of food deprivation with access to water ad libitum. The deprivation period started after 4 hours of inversion of the light / dark cycle. After the controlled period of deprivation, all animals were subjected to the collection of a drop of blood from the caudal vein and the measurement was performed immediately using the Accu-Chek Performa glucometer (ROCHE).

\section{Statistics analysis}

Data were analyzed using GraphPad Prism 8.0.1 (GraphPad Software Inc.,CA, USA). The statistical analyses were carried out using unpaired two-tailed Student's t-test, two-way analysis of variance (ANOVA) or ANOVA with repeated measures when appropriate. Post hoc comparisons were performed using the Bonferroni test. Data are presented as means \pm standard error of the mean (SEM). A p value < 0.05 was considered to be statistically significant.

\section{Results}

\section{Coumarin treatment reduces fasting blood glucose levels}

Body weight was measured weekly, while fasting blood glucose was measured on the last day of the experimental protocol. Blood samples were obtained by caudal puncture. Statistical analysis using twoway ANOVA, being an experimental group (vehicle $x$ DHMC) $X$ time interaction (weight gain), showed that there was no significant difference in body weight between the groups (Fig. $1 \mathrm{~b})[F(3,140)=1,641$; $p=0.1827]$, but a significant main effect of time $[F(4,140)=33.93 ; p=0.0001]$ reflecting a change in weight, regardless of treatment (vehicle or DHMC). However, fasting blood glucose levels were reduced in animals treated with DHMC, for both sedentary and voluntary physical activity groups (Fig.1c) [F(1, $28)=4.515 ; p=0.0426$ ]. To investigate the effect of DMHC in isolation, we used a paired test (vehicle $x$ DMHC) considering all animals, exercised or not from each group (Fig.1d). The animals supplemented with $\mathrm{DHMC}$ showed a significant reduction in fasting glycemia when compared to their vehicle controls $[\mathrm{t}$ $=2,158, \mathrm{df}=30 ; \mathrm{p}=0.0391]$.

\section{Effect of coumarin treatment and physical activity in anxiolytic behavior}

To determine the effects of voluntary physical activity and DHMC on behavioral responses related to anxiety, the rats underwent two interventions. Briefly, the amount of daily physical activity was obtained, which corresponds to the total number of complete rotations and volume performed in the light and dark periods of the day. The physical activity group was monitored through daily records, obtained from the voluntary physical activity wheel, and the sedentary group was not exposed to the equipment. The data were initially submitted to two-way ANOVA (vehicle x DHMC) X revolutions per minute per day (RPM/day) 
over the 29 days of the experiment. We found that DHMC administration decreased the total volume of physical activity in the light period (Fig.2a) $[F(1,286)=9.711 ; p=0.0020]$ and confirmed by the analysis of the total volume by the paired t-test, considering the sum of the 29 experimental days (Fig.2c) [t=2.861, $d f=10 ; p=0.0169]$. Interestingly, no differences were identified during the dark period (Fig.2b) $[F(28,338)=$ 4.610; $p<0.1781]$ and (Fig.2d) $[t=0.08443, d f=13 ; p=0.9340]$. In a second approach, the mice's locomotion index and anxiolytic response were assessed in the EPM task. As expected, the physical activity group showed an increased locomotion index, considering the total number of entries in all arms of the labyrinth ( $F i g .2 e)[F(1,16)=22.15 ; p=0.0002]$. In addition, physical activity reduced the animals' anxious behavior, characterized by a longer stay in the open arms of the labyrinth (Fig.2f) $[F(1,16)=22.76$; $p=0.0002]$, although there was no difference in the percentage of entries between the open and closed arms in relation to the total number of both arms (Fig.2g) $[F(1,16)=0.02631 ; p=0.8732]$.

\section{Coumarin treatment increases hypothalamic VEGF gene expression}

To evaluate the neurotrophic pathways that could be activated by treatment with coumarin or physical activity, the hypothalamic tissue was prepared for analysis of gene expression (Fig.3). We used bidirectional ANOVA considering the treatment (vehicle $x$ DHMC) $X$ neurotrophic markers (fold change). We observed a higher average gene expression of DCX, CNTF, BDNF and VEGF (Fig.3a, c, e and f) in groups treated with coumarin, respectively $[F(1,12)=0.3550 ; p=0.5624],[F(1,12)=0.2763 ; p=0.6087]$, $[F(1,12)=0.2212 ; p=0.6466]$ and $[F(1,12)=0.8718 ; p=0.3689]$, although our number of replicates only allows for a statistical difference when comparing sedentary vehicles and groups treated with coumarin for VEGF expression by means of a paired $t$ test considering animals exercised or not together (Fig.3g) $[t=3.094, d f=6 ; p=0.0213]$. The NEUROD1 mRNA levels (Fig. 3B) $[F(1,12)=0.001866 ; p=$ $0.9663]$ and CNFTR (Fig.3d) $[F(1,12)=1,205 ; p=0.2940]$ remained unchanged. Interestingly, we found a positive correlation when comparing VEGF expression and fasting glucose levels (Fig.3h) $[F=6,802 ; R=$ $0.3270 p=0.0207]$.

\section{Discussion}

The overall increase in life expectancy has been accompanied by growing incidence of aging-related neuropsychiatric conditions such as Alzheimer and Parkinson's diseases, depression and anxiety (Armstrong and Okun 2020; Curran et al. 2020). Throughout life the complex central nervous system environment seems to depend on a balance of factors to maintain optimal neuronal function (Myers and Olson 2012). Among the various strategies on debate to prevent, delay or reverse neurodegeneration development, systemic approaches, such as physical activity, and new pharmacological molecules outstand from classic interventions, given their lower potential for adverse effects (Chen and Shan 2019; Bhatti et al. 2020). In this study, we assessed the effects of voluntary physical activity and DHMC, independently or in combination, over physiological and behavioral parameters, as well as an investigation of their neuroplasticity effects in the hypothalamus and hippocampus at a molecular and cellular level. 
First, we asked if physical activity would produce anxiolytic-like behavior and the potential incremental effect of DHMC treatment. Physical activity comprehends body movements produced by skeletal muscles that results in energy expenditure and it is positively correlated with physical fitness (Caspersen et al. 1985). Mounting data have shown that physical activity/exercise interventions promote improvements in chronic diseases such as metabolic, cardiovascular and neurodegenerative diseases (Angevaren et al. 2008; Shapiro et al. 2011; Spartano et al. 2017). Additionally, it is remarkable the effectiveness of physical activity in improving anxiety symptoms in people with a current diagnosis of anxiety and/ or stress-related disorders (Kandola et al. 2018). At the preclinical level, the voluntary running wheel is a useful method of increasing physical activity in rodents (Cunha et al. 2013; Alkadhi 2018). As expected, we observed an anxiolytic effect of physical activity evidenced by an increased time spent in the open arms in the EPM, corroborating previous studies (Hötting and Röder 2013; Caliskan et al. 2019). The circadian clock and inadequate rest time can influence several processes involved in neurodegeneration and morphological and functional changes in the neuronal environment (Musiek and Holtzman 2016). Interestingly, the DHMC treatment produced an additional anxiolytic effect in the physical activity group, observed as a decreased volume of voluntary physical activity during the light cycle (rest period for rodents). In concert, it was previously reported that DHMC alleviated chronic unpredictable mild stress-induced depression-like behaviors and alterations in spine density in rats (Yang et al. 2020). However, we recognize that the anxiolytic effect of DHMC was only found in one of its measures (voluntary activity wheel) and other models of behavioral experiments could be considered in the future.

Next, we investigated if our interventions were able to induce neuroplasticity alterations. In animal models, physical activity mitigates age related impairment in adult neurogenesis and cognition in the hippocampus, preserving functions such as learning, memory and emotional behavior (Praag et al. 2005; Hill et al. 2015). Although we confirmed the anxiolytic effect of physical activity and a further action of DHMC, in our experimental conditions, they were not accompanied by an increment in adult hippocampal or hypothalamic neurogenesis. Adult neurogenesis is the process that leads to the formation of functional new neurons, in certain regions of the adult brain, such as the subgranular zone of the dentate gyrus (DG), subventricular zone of the lateral ventricles and, at a lower rate, at the ventricular zone of the hypothalamus (Ming and Song 2011). Adult born neurons integrate the established circuitry and modulate structure-related functions, for instance cognition and emotional behavior in the DG (Sahay and Hen 2008; Deng et al. 2010), and body energy homeostasis in the hypothalamus (Kokoeva 2005a).

The mice hypothalamus presents some neurogenic activity during adulthood, which has been reported to play a role in long-term metabolic control (Kokoeva 2005b; Lee et al. 2012; Li et al. 2012). Hypothalamic neurons are critical sensors of nutrient availability implicated in energy balance and glucose metabolism control (Schneeberger et al. 2014). While we could not see differences in adult hypothalamic neurogenesis, we found that DHMC produced a reduction in fasting glucose, a positive metabolic feature when considering the risk for development of metabolic conditions (Wilson 2017). Similar improvements in glucose metabolism have been described for other methyl coumarins that particularly enhanced insulin secretion (Ahmed et al. 2020). Of note, metabolic disorders are also correlated to a higher risk of 
developing neuropsychiatric conditions (Bădescu et al. 2016; Lyra e Silva et al. 2019), which makes DHMC an interesting candidate to be assessed as a new pharmacological approach in the association of metabolic and neurodegenerative disorders. Of note, an important limitation of our study is that, despite the sex differences in neurodegenerative and metabolic-related disorders, we used only male mice. Hence, the data should be amplified in female mice for a more translational interpretation. Additionally, although here we focused on a preventive strategy, another future perspective would be to challenge the animals with neurodegenerative insults (such as aging, chronic stress, high-fat diet exposure), which could reveal a stronger effect of the association of DHMC and physical activity, especially in neuroplasticity assessments.

Although limbic structures are the major sites comprehending the neural circuitry regulating behavioral outcomes in mood disorders (Price and Drevets 2010), dysfunction in the hypothalamus-pituitary-adrenal (HPA) axis is commonly known to be associated with stress-related development of behavioral conditions, such as anxiety and depression (Kessing et al. 2011; Russell and Lightman 2019). In this scenario, important findings mediating cellular neurodegeneration are the impairment of neuroplasticity and neurotrophic factors supply (Duman et al. 1997; Alleva and Francia 2009). Among the neurotrophins that are important regulators of neural survival, development, function, and plasticity are BDNF, CNTF, VEGF and others (Duman 2009; Fargali et al. 2012). In addition to the widely studied hippocampal related neurotrophic activity, several studies have uncovered a central role for hypothalamic neurotrophins, specially BDNF, upon multiple circuits to govern appetite and energy metabolism (Xu et al. 2003; Kokoeva 2005b; An et al. 2020). Therefore, it is known that behavioral disturbances causing derangement in the HPA axis are, as well, correlated to metabolic outcomes(Ivić et al. 2016; Jelenik et al. 2018). It was previously shown that chronically stressed rats presented reduced hypothalamic and pituitary VEGF and BDNF mRNA levels (Nowacka et al. 2015). Conversely, in our study, we found that DHMC treatment increased hypothalamic VEGF gene expression, which was also positively correlated with reduced fasting glucose levels. In this regard, Langlet and colleagues demonstrated an important role of tanycytic VEGF in blood-hypothalamus barrier plasticity and hypothalamic metabolic response to fasting (Langlet et al. 2013) corroborating the correlation found in our study. Moreover, it has been shown that VEGF regulates GLUT-1 expression and glucose uptake in blood brain barrier cells of obese mice, and this VEGF mediated homeostatic regulation limits cognitive impaiment in obesity models (Jais et al. 2016). Supporting this line of experimental evidence, in humans, high fat diet consumption modulated tissue expression of GLUT1 and serum VEGF levels, which was correlated to performance in cognitive tests (Schüler et al. 2018).

\section{Conclusions}

In conclusion, our data reinforce the positive outcomes of physical activity in promoting neuroplasticity improvements that could mitigate the development of neuropsychiatric conditions, such as anxiety, and bring up DHMC as a potentially useful pharmacological approach that could be assessed in further studies targeting neurodegenerative conditions associated with metabolic disorders. 


\section{Declarations}

\section{Author contributions}

PKFL, DFE, MSAM, NOB and CAP performed experiments and analyzed the data; DFE and PKFL wrote the manuscript; RFM designed the study; all authors revised and approved the final version of the manuscript.

\section{Acknowledgement}

We thank the access to equipment and assistance provided by the National Institute of Science and Technology on Photonics Applied to Cell Biology (INFABIC) at the State University of Campinas.

\section{Funding}

The study was supported by Grants from the Minas Gerais Research Support Foundation (FAPEMIG), Conselho Nacional de Pesquisa e Desenvolvimento Cientifico (CNPq), São Paulo Research Foundation (2013/07607-8), and financed in part by the Coordenação de Aperfeiçoamento de Pessoal de Nível Superior - Brasil (CAPES); INFABIC is co-funded by São Paulo Research Foundation (FAPESP) (2014/50938-8) and Conselho Nacional de Desenvolvimento Cientifico e Tecnológico (CNPq) (465699/2014-6).

\section{Conflicts of interest}

The authors have no conflicts of interest to declare that are relevant to the content of this article.

\section{Availability of data and material}

The datasets generated during and/or analyzed during the current study are available from the corresponding author on reasonable request.

\section{Ethics approval}

All experimental procedures were carried out in accordance with the National Institutes of Health Guide for the Care and Use of Laboratory Animals, with the approval of the Ethics Committee of the Federal University of Lavras/Brazil and Animal Experiments Control Council (CONCEA), according to protocol $\mathrm{n}^{\circ}$ 078/17.

\section{References}

1. Ahmed S, Nur-e-Alam M, Parveen I, et al (2020) Stimulation of insulin secretion by 5methylcoumarins and its sulfur analogues isolated from Clutia lanceolata Forssk. Phytochemistry 170:112213. https://doi.org/10.1016/j.phytochem.2019.112213 
2. Alkadhi KA (2018) Exercise as a Positive Modulator of Brain Function. Mol Neurobiol 55:3112-3130. https://doi.org/10.1007/s12035-017-0516-4

3. Alleva E, Francia N (2009) Psychiatric vulnerability: Suggestions from animal models and role of neurotrophins. Neuroscience \& Biobehavioral Reviews 33:525-536. https://doi.org/10.1016/j.neubiorev.2008.09.004

4. An JJ, Kinney CE, Tan J-W, et al (2020) TrkB-expressing paraventricular hypothalamic neurons suppress appetite through multiple neurocircuits. Nature Communications 11:1729. https://doi.org/10.1038/s41467-020-15537-w

5. Angevaren M, Aufdemkampe G, Verhaar HJJ, et al (2008) Physical activity and enhanced fitness to improve cognitive function in older people without known cognitive impairment. Cochrane Database of Systematic Reviews. https://doi.org/10.1002/14651858.CD005381.pub3

6. Armstrong MJ, Okun MS (2020) Diagnosis and Treatment of Parkinson Disease: A Review. JAMA 323:548-560. https://doi.org/10.1001/jama.2019.22360

7. Bădescu S, Tătaru C, Kobylinska L, et al (2016) The association between Diabetes mellitus and Depression. J Med Life 9:120-125

8. Bhatti GK, Reddy AP, Reddy PH, Bhatti JS (2020) Lifestyle Modifications and Nutritional Interventions in Aging-Associated Cognitive Decline and Alzheimer's Disease. Front Aging Neurosci 11:. https://doi.org/10.3389/fnagi.2019.00369

9. Caliskan H, Akat F, Tatar Y, et al (2019) Effects of exercise training on anxiety in diabetic rats. Behavioural Brain Research 376:112084. https://doi.org/10.1016/j.bbr.2019.112084

10. Carrera I, Martínez O, Cacabelos R (2020) Neuroprotection with Natural Antioxidants and Nutraceuticals in the Context of Brain Cell Degeneration: The Epigenetic Connection. CTMC 19:2999-3011. https://doi.org/10.2174/1568026619666191202155738

11. Caspersen CJ, Powell KE, Christenson GM (1985) Physical activity, exercise, and physical fitness: definitions and distinctions for health-related research. Public Health Rep 100:126-131

12. Chen C, Shan W (2019) Pharmacological and non-pharmacological treatments for major depressive disorder in adults: A systematic review and network meta-analysis. Psychiatry Research 281:112595. https://doi.org/10.1016/j.psychres.2019.112595

13. Choi SH, Bylykbashi E, Chatila ZK, et al (2018) Combined adult neurogenesis and BDNF mimic exercise effects on cognition in an Alzheimer's mouse model. Science 361:eaan8821. https://doi.org/10.1126/science.aan8821

14. Cotman CW, Berchtold NC, Christie L-A (2007) Exercise builds brain health: key roles of growth factor cascades and inflammation. Trends in Neurosciences 30:464-472. https://doi.org/10.1016/j.tins.2007.06.011

15. Cunha MP, Oliveira Á, Pazini FL, et al (2013) The Antidepressant-like Effect of Physical Activity on a Voluntary Running Wheel. Medicine \& Science in Sports \& Exercise 45:851-859. https://doi.org/10.1249/MSS.0b013e31827b23e6 
16. Curran E, Rosato M, Ferry F, Leavey G (2020) Prevalence and factors associated with anxiety and depression in older adults: Gender differences in psychosocial indicators. Journal of Affective Disorders 267:114-122. https://doi.org/10.1016/j.jad.2020.02.018

17. Deng W, Aimone JB, Gage FH (2010) New neurons and new memories: how does adult hippocampal neurogenesis affect learning and memory? Nature Reviews Neuroscience 11:339-350. https://doi.org/10.1038/nrn2822

18. Dugger BN, Dickson DW (2017) Pathology of Neurodegenerative Diseases. Cold Spring Harb Perspect Biol 9:a028035. https://doi.org/10.1101/cshperspect.a028035

19. Duman RS (2009) Neuronal damage and protection in the pathophysiology and treatment of psychiatric illness: stress and depression. Dialogues Clin Neurosci 11:239-255

20. Duman RS, Heninger GR, Nestler EJ (1997) A Molecular and Cellular Theory of Depression. Arch Gen Psychiatry 54:597-606. https://doi.org/10.1001/archpsyc.1997.01830190015002

21. Estrada JA, Contreras I (2019) Nutritional Modulation of Immune and Central Nervous System Homeostasis: The Role of Diet in Development of Neuroinflammation and Neurological Disease. Nutrients 11:1076. https://doi.org/10.3390/nu11051076

22. Fabel K, Fabel K, Tam B, et al (2003) VEGF is necessary for exercise-induced adult hippocampal neurogenesis. European Journal of Neuroscience 18:2803-2812. https://doi.org/10.1111/j.14609568.2003.03041.x

23. Fargali S, Sadahiro M, Jiang C, et al (2012) Role of Neurotrophins in the Development and Function of Neural Circuits That Regulate Energy Homeostasis. J Mol Neurosci 48:654-659. https://doi.org/10.1007/s12031-012-9790-9

24. Gao Q, Jeon SJ, Jung HA, et al (2015) Nodakenin Enhances Cognitive Function and Adult Hippocampal Neurogenesis in Mice. Neurochem Res 40:1438-1447. https://doi.org/10.1007/s11064-015-1612-3

25. Gao Z, Wen Q, Xia Y, et al (2014) Osthole augments therapeutic efficiency of neural stem cells-based therapy in experimental autoimmune encephalomyelitis. J Pharmacol Sci 124:54-65. https://doi.org/10.1254/jphs.13144fp

26. Hill AS, Sahay A, Hen R (2015) Increasing Adult Hippocampal Neurogenesis is Sufficient to Reduce Anxiety and Depression-Like Behaviors. Neuropsychopharmacology 40:2368-2378. https://doi.org/10.1038/npp.2015.85

27. Hötting K, Röder B (2013) Beneficial effects of physical exercise on neuroplasticity and cognition. Neuroscience \& Biobehavioral Reviews 37:2243-2257. https://doi.org/10.1016/j.neubiorev.2013.04.005

28. Ivić V, Blažetić S, Labak I, et al (2016) Ovariectomy and chronic stress lead toward leptin resistance in the satiety centers and insulin resistance in the hippocampus of Sprague-Dawley rats. Croat Med $\mathrm{J}$ 57:194-206. https://doi.org/10.3325/cmj.2016.57.194

29. Jais A, Solas M, Backes H, et al (2016) Myeloid-Cell-Derived VEGF Maintains Brain Glucose Uptake and Limits Cognitive Impairment in Obesity. Cell 165:882-895. 
https://doi.org/10.1016/j.cell.2016.03.033

30. Jameel E, Umar T, Kumar J, Hoda N (2016) Coumarin: A Privileged Scaffold for the Design and Development of Antineurodegenerative Agents. Chemical Biology \& Drug Design 87:21-38. https://doi.org/10.1111/cbdd.12629

31. Jelenik T, Dille M, Müller-Lühlhoff S, et al (2018) FGF21 regulates insulin sensitivity following longterm chronic stress. Molecular Metabolism 16:126-138.

https://doi.org/10.1016/j.molmet.2018.06.012

32. Jin X, Wang Y, Li X, et al (2015) 7,8-Dihydroxy-4-methylcoumarin Provides Neuroprotection by Increasing Hippocalcin Expression. Neurotox Res 27:268-274. https://doi.org/10.1007/s12640-0149507-7

33. Kadakol A, Sharma N, Kulkarni YA, Gaikwad AB (2016) Esculetin: A phytochemical endeavor fortifying effect against non-communicable diseases. Biomedicine \& Pharmacotherapy 84:14421448. https://doi.org/10.1016/j.biopha.2016.10.072

34. Kandola A, Vancampfort D, Herring M, et al (2018) Moving to Beat Anxiety: Epidemiology and Therapeutic Issues with Physical Activity for Anxiety. Curr Psychiatry Rep 20:63. https://doi.org/10.1007/s11920-018-0923-x

35. Kang SY, Kim YC (2007) Decursinol and decursin protect primary cultured rat cortical cells from glutamate-induced neurotoxicity. Journal of Pharmacy and Pharmacology 59:863-870. https://doi.org/10.1211/jpp.59.6.0013

36. Kessing LV, Willer IS, Knorr U (2011) Volume of the adrenal and pituitary glands in depression. Psychoneuroendocrinology 36:19-27. https://doi.org/10.1016/j.psyneuen.2010.05.007

37. Kokoeva MV (2005a) Neurogenesis in the Hypothalamus of Adult Mice: Potential Role in Energy Balance. Science 310:679-683. https://doi.org/10.1126/science.1115360

38. Kokoeva MV (2005b) Neurogenesis in the Hypothalamus of Adult Mice: Potential Role in Energy Balance. Science 310:679-683. https://doi.org/10.1126/science.1115360

39. Kokoeva MV, Yin H, Flier JS (2007) Evidence for constitutive neural cell proliferation in the adult murine hypothalamus. J Comp Neurol 505:209-220. https://doi.org/10.1002/cne.21492

40. Kronenberg G, Bick-Sander A, Bunk E, et al (2006) Physical exercise prevents age-related decline in precursor cell activity in the mouse dentate gyrus. Neurobiology of Aging 27:1505-1513. https://doi.org/10.1016/j.neurobiolaging.2005.09.016

41. Langlet F, Levin BE, Luquet $S$, et al (2013) Tanycytic VEGF-A boosts blood-hypothalamus barrier plasticity and access of metabolic signals to the arcuate nucleus in response to fasting. Cell Metab 17:607-617. https://doi.org/10.1016/j.cmet.2013.03.004

42. Lee DA, Bedont JL, Pak T, et al (2012) Tanycytes of the hypothalamic median eminence form a dietresponsive neurogenic niche. Nat Neurosci 15:700-702. https://doi.org/10.1038/nn.3079

43. Li J, Tang Y, Cai D (2012) IKKB/NF-KB disrupts adult hypothalamic neural stem cells to mediate a neurodegenerative mechanism of dietary obesity and pre-diabetes. Nature Cell Biology 14:999-1012. https://doi.org/10.1038/ncb2562 
44. Lyra e Silva N de M, Gonçalves RA, Boehnke SE, et al (2019) Understanding the link between insulin resistance and Alzheimer's disease: Insights from animal models. Experimental Neurology 316:1-11. https://doi.org/10.1016/j.expneurol.2019.03.016

45. Ming G, Song H (2011) Adult Neurogenesis in the Mammalian Brain: Significant Answers and Significant Questions. Neuron 70:687-702. https://doi.org/10.1016/j.neuron.2011.05.001

46. Molina-Jiménez MF, Sánchez-Reus MI, Cascales M, et al (2005) Effect of fraxetin on antioxidant defense and stress proteins in human neuroblastoma cell model of rotenone neurotoxicity. Comparative study with myricetin and $\mathrm{N}$-acetylcysteine. Toxicology and Applied Pharmacology 209:214-225. https://doi.org/10.1016/j.taap.2005.04.009

47. Musiek ES, Holtzman DM (2016) Mechanisms linking circadian clocks, sleep, and neurodegeneration. Science 354:1004-1008. https://doi.org/10.1126/science.aah4968

48. Myers MG, Olson DP (2012) Central nervous system control of metabolism. Nature 491:357-363. https://doi.org/10.1038/nature11705

49. Niwa A, Nishibori M, Hamasaki S, et al (2016) Voluntary exercise induces neurogenesis in the hypothalamus and ependymal lining of the third ventricle. Brain Struct Funct 221:1653-1666. https://doi.org/10.1007/s00429-015-0995-x

50. Nowacka MM, Paul-Samojedny M, Bielecka AM, et al (2015) LPS reduces BDNF and VEGF expression in the structures of the HPA axis of chronic social stressed female rats. Neuropeptides 54:17-27. https://doi.org/10.1016/j.npep.2015.09.003

51. Potdar MK, Mohile SS, Salunkhe MM (2001) Coumarin syntheses via Pechmann condensation in Lewis acidic chloroaluminate ionic liquid. Tetrahedron Letters 42:9285-9287. https://doi.org/10.1016/S0040-4039(01)02041-X

52. Praag H van, Shubert T, Zhao C, Gage FH (2005) Exercise Enhances Learning and Hippocampal Neurogenesis in Aged Mice. J Neurosci 25:8680-8685. https://doi.org/10.1523/JNEUROSCI.173105.2005

53. Price JL, Drevets WC (2010) Neurocircuitry of Mood Disorders. Neuropsychopharmacology 35:192216. https://doi.org/10.1038/npp.2009.104

54. Qin T, Fang F, Song M, et al (2017) Umbelliferone reverses depression-like behavior in chronic unpredictable mild stress-induced rats by attenuating neuronal apoptosis via regulating ROCK/Akt pathway. Behavioural Brain Research 317:147-156. https://doi.org/10.1016/j.bbr.2016.09.039

55. Rajadurai M, Stanely Mainzen Prince P (2006) Preventive effect of naringin on lipid peroxides and antioxidants in isoproterenol-induced cardiotoxicity in Wistar rats: Biochemical and histopathological evidences. Toxicology 228:259-268. https://doi.org/10.1016/j.tox.2006.09.005

56. Russell G, Lightman S (2019) The human stress response. Nature Reviews Endocrinology 15:525534. https://doi.org/10.1038/s41574-019-0228-0

57. Sahay A, Hen R (2008) Hippocampal neurogenesis and depression. Novartis Found Symp 289:152160; discussion 160-164, 193-195 
58. Santarelli L, Saxe M, Gross C, et al (2003) Requirement of hippocampal neurogenesis for the behavioral effects of antidepressants. Science 301:805-809.

https://doi.org/10.1126/science.1083328

59. Schneeberger M, Gomis R, Claret M (2014) Hypothalamic and brainstem neuronal circuits controlling homeostatic energy balance. Journal of Endocrinology 220:T25-T46. https://doi.org/10.1530/JOE13-0398

60. Schüler R, Seebeck N, Osterhoff MA, et al (2018) VEGF and GLUT1 are highly heritable, inversely correlated and affected by dietary fat intake: Consequences for cognitive function in humans. Molecular Metabolism 11:129-136. https://doi.org/10.1016/j.molmet.2018.02.004

61. Shapiro A, Cheng K-Y, Gao Y, et al (2011) The Act of Voluntary Wheel Running Reverses Dietary Hyperphagia and Increases Leptin Signaling in Ventral Tegmental Area of Aged Obese Rats. GER 57:335-342. https://doi.org/10.1159/000321343

62. Skalicka-Woźniak K, Orhan IE, Cordell GA, et al (2016) Implication of coumarins towards central nervous system disorders. Pharmacological Research 103:188-203.

https://doi.org/10.1016/j.phrs.2015.11.023

63. Spartano NL, Stevenson MD, Xanthakis V, et al (2017) Associations of objective physical activity with insulin sensitivity and circulating adipokine profile: the Framingham Heart Study. Clinical Obesity 7:59-69. https://doi.org/10.1111/cob.12177

64. Swift DL, McGee JE, Earnest CP, et al (2018) The Effects of Exercise and Physical Activity on Weight Loss and Maintenance. Progress in Cardiovascular Diseases 61:206-213. https://doi.org/10.1016/j.pcad.2018.07.014

65. Togna AR, Firuzi O, Latina V, et al (2014) 4-Methylcoumarin derivatives with anti-inflammatory effects in activated microglial cells. Biol Pharm Bull 37:60-66. https://doi.org/10.1248/bpb.b13-00568

66. Tyagi YK, Kumar A, Raj HG, et al (2005) Synthesis of novel amino and acetyl amino-4methylcoumarins and evaluation of their antioxidant activity. European Journal of Medicinal Chemistry 40:413-420. https://doi.org/10.1016/j.ejmech.2004.09.002

67. van Praag H, Kempermann G, Gage FH (1999) Running increases cell proliferation and neurogenesis in the adult mouse dentate gyrus. Nat Neurosci 2:266-270. https://doi.org/10.1038/6368

68. Wilson ML (2017) Prediabetes: Beyond the Borderline. Nurs Clin North Am 52:665-677. https://doi.org/10.1016/j.cnur.2017.07.011

69. Wu L, Wang X, Xu W, et al (2009) The structure and pharmacological functions of coumarins and their derivatives. Curr Med Chem 16:4236-4260. https://doi.org/10.2174/092986709789578187

70. Xu B, Goulding EH, Zang K, et al (2003) Brain-derived neurotrophic factor regulates energy balance downstream of melanocortin-4 receptor. Nature Neuroscience 6:736-742. https://doi.org/10.1038/nn1073

71. Yang M, Luo C-H, Zhu Y-Q, et al (2020) 7, 8-Dihydroxy-4-methylcoumarin reverses depression modelinduced depression-like behaviors and alteration of dendritic spines in the mood circuits. Psychoneuroendocrinology 119:104767. https://doi.org/10.1016/j.psyneuen.2020.104767 
72. Yao Y, Gao Z, Liang W, et al (2015) Osthole promotes neuronal differentiation and inhibits apoptosis via Wnt/ $\beta$-catenin signaling in an Alzheimer's disease model. Toxicology and Applied Pharmacology 289:474-481. https://doi.org/10.1016/j.taap.2015.10.013

\section{Figures}

a)

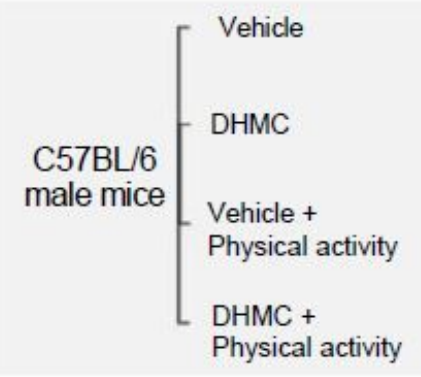

b)

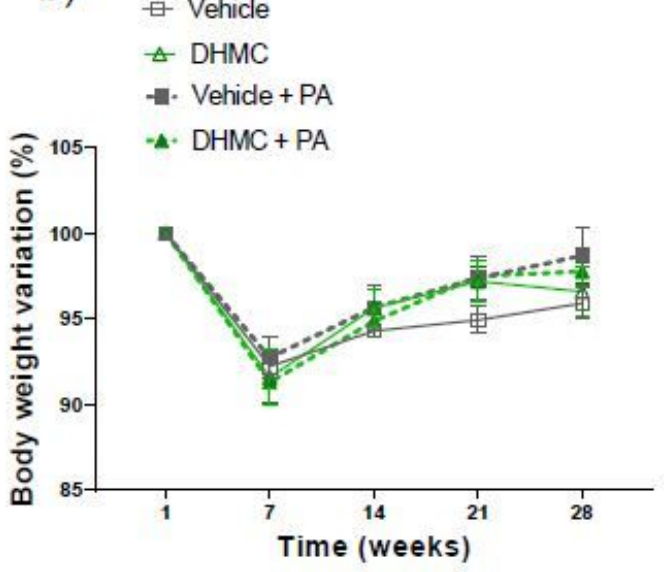

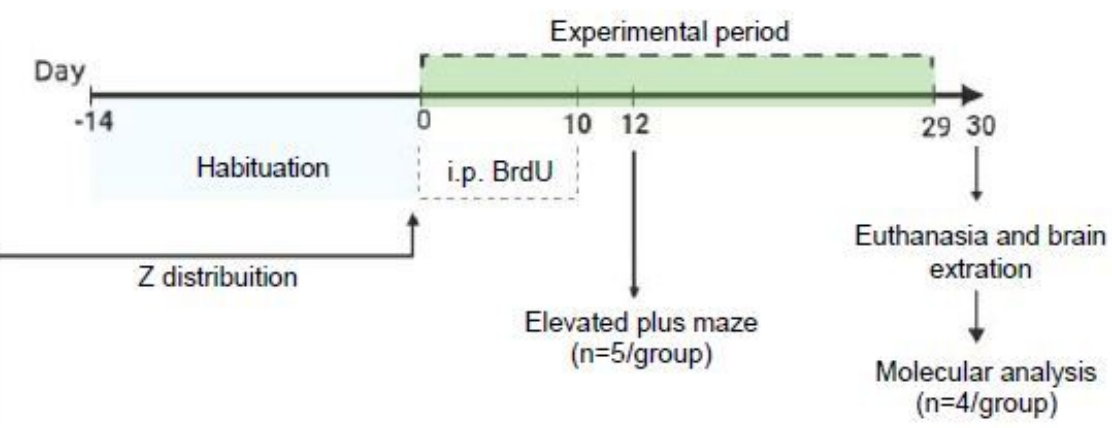

c)

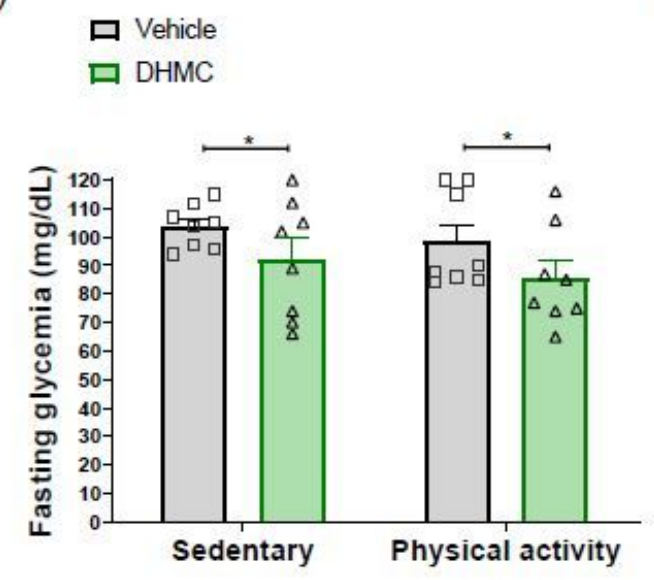

d)

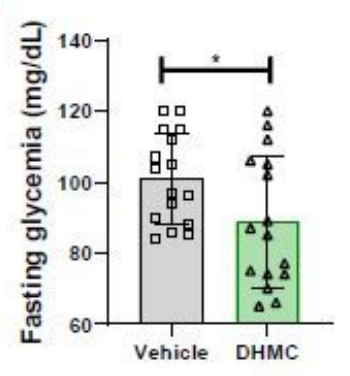

\section{Figure 1}

DHMC reduced fasting blood glucose. Panel A shows the experimental design. C57BL/6J mice received a 29-day repeated treatment of 7,8-Dihydroxy-4-methylcoumarin (DHMC) or vehicle i.g. In the first 10-day mice received BrdU (5-Bromo-2'-deoxyuridine) 50mg/wt, 2x/day i.p.,12/12 hours. Part of the animals were exposed to the voluntary physical activity wheel during the whole experiment (physical activity groups) while the others did not have access to the wheel (sedentary groups) ( $n=8$ per group). On the 12th day the mice were exposed to elevated plus maze paradigm (EPM) in order to assess anxiety-related behaviors ( $n=5$ per group). The animals were sacrificed 20 days after the last BrdU injection by transcardial perfusion and their brains were processed for molecular analyses. (B) Anova two-way weekly bodyweight gain (vehicle $x$ DHMC) $X$ time interaction (weight gain) (C) Anova two-way fasting blood glucose levels (vehicle $x$ DHMC) $X$ fasting glycemia $(\mathrm{mg} / \mathrm{dL}$ ) (D) Paired t test (vehicle $x$ DMHC). Statistical significance 
defined when ${ }^{*} \mathrm{p}<0.05$. Data are presented as means + SEM. Kolmogorov-Smirnov normality and variance equality F-test. DHMC= 7,8-dihydroxy-4-methylcoumarin; BrdU= 5-bromo-2'-deoxyuridine

a)

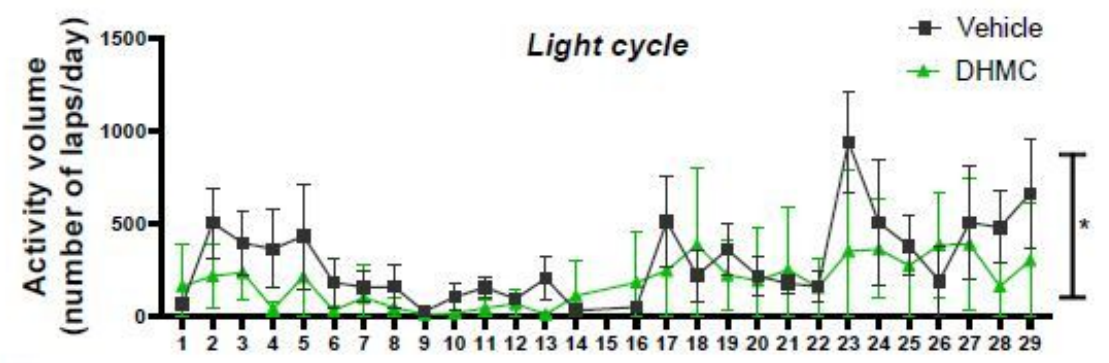

b)

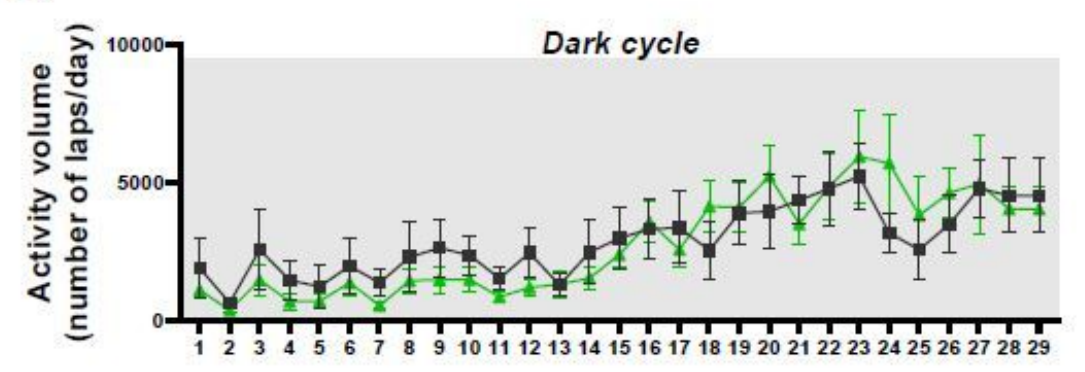

e)

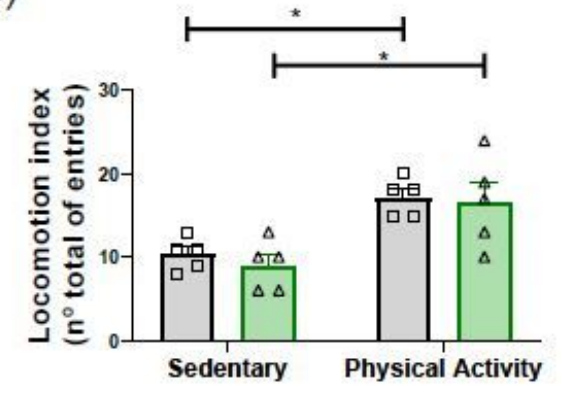

f)

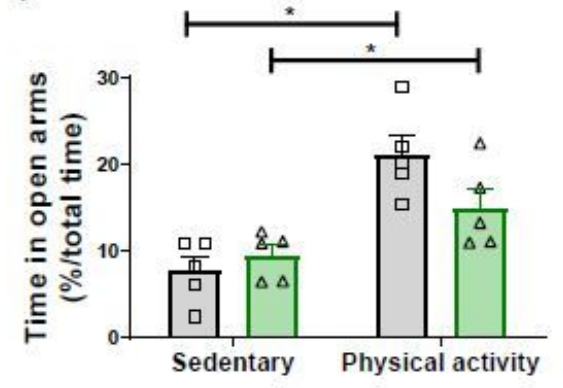

c)

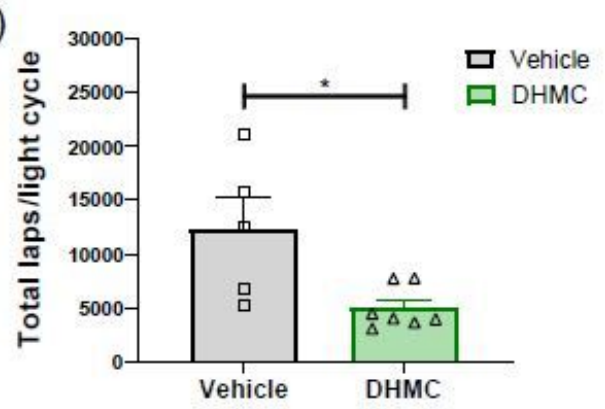

d)

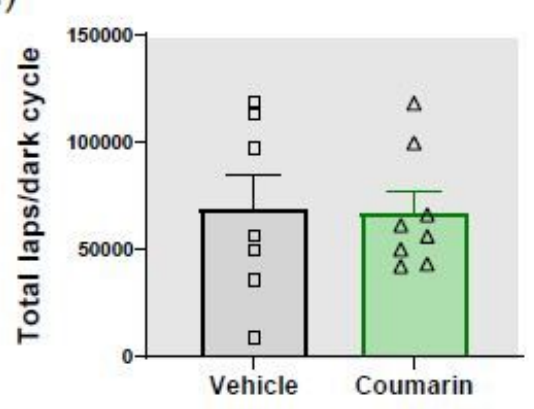

g)

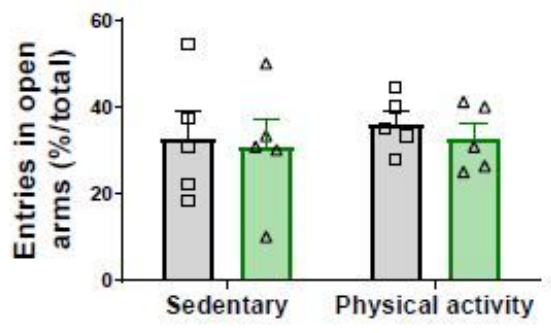

\section{Figure 2}

Effect of DHMC treatment and physical activity on anxiolytic behavior. (A) Two-way ANOVA (vehicle $x$ DHMC) X revolutions per minute per day (RPM/day) over the 29 days of the experiment for daily volume of voluntary physical activity during the light or (B) Paired t test (vehicle $x$ DMHC) for total volume in the dark cycle. (C) Two-way ANOVA (vehicle $x$ DHMC) X revolutions per minute per day (RPM/day) over the 29 days of the experiment for total volume of voluntary physical activity during the light or (D) Paired $t$ test (vehicle $x$ DMHC) for total volume in the dark cycle (E) Two-way ANOVA (vehicle $x$ DHMC) $X$ locomotion index (total number of entries) for locomotion index obtained by the total number of entries in the open and closed arms (F) Two-way ANOVA (vehicle $x$ DHMC) $X$ time in open arms (\%/total time) for total time in the open arms and (G) Two-way ANOVA (vehicle $x$ DHMC) $X$ entries in open arms (\%/total) for number of entries in the open arms; both represented as a percentage of total entries in both arms. Data are presented as means $+\mathrm{SEM}$. $\mathrm{N}=8$ per group for analyzes related to the voluntary physical activity wheel (A-D) and $N=5$ per group for analyzes related to the elevated plus maze test (E-G).Statistical significance defined when ${ }^{*} p<0.05$. Data are presented as means $+S E M$. Kolmogorov-Smirnov normality and variance equality F-test. $\mathrm{DHMC}=7,8$-dihydroxy-4-methylcoumarin 
a)

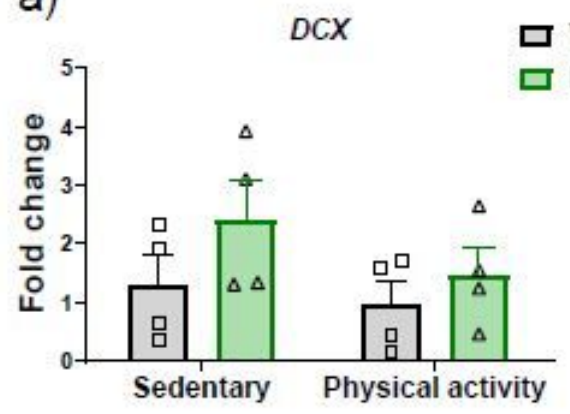

c)

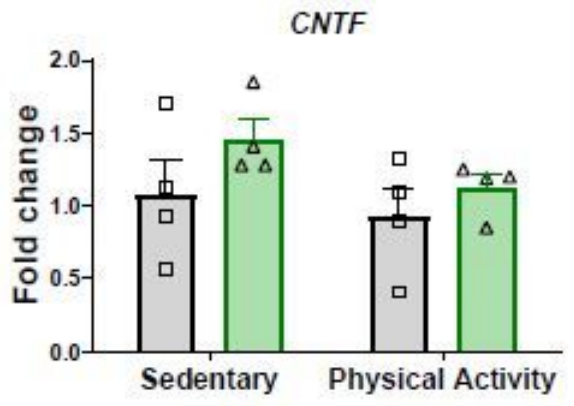

e)

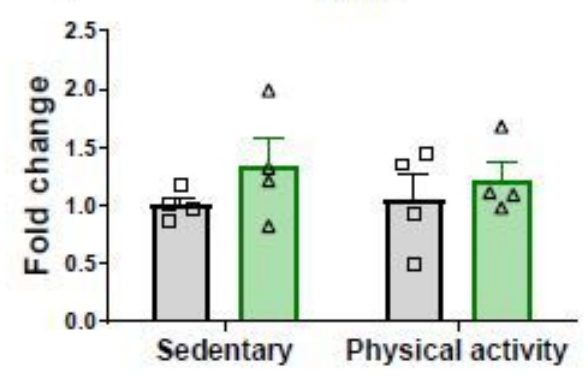

g)

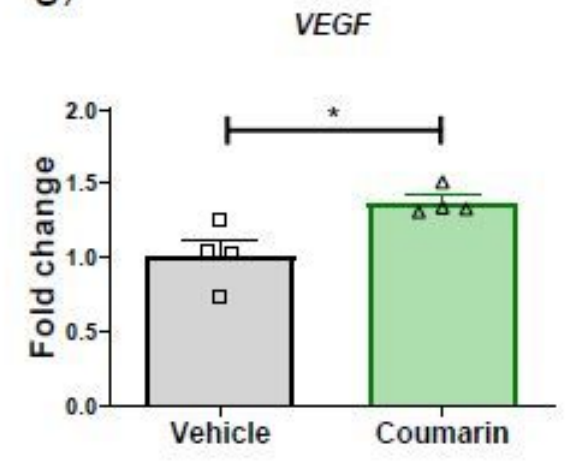

b) DHMC

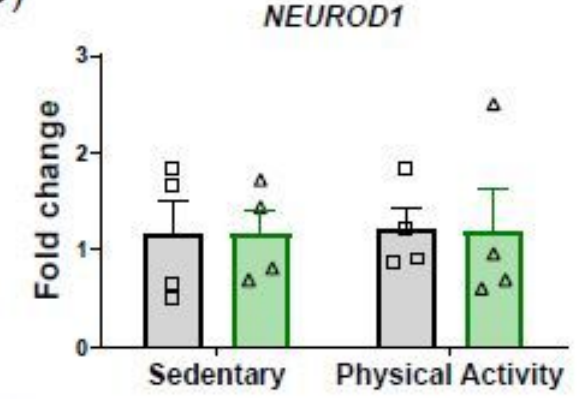

d)

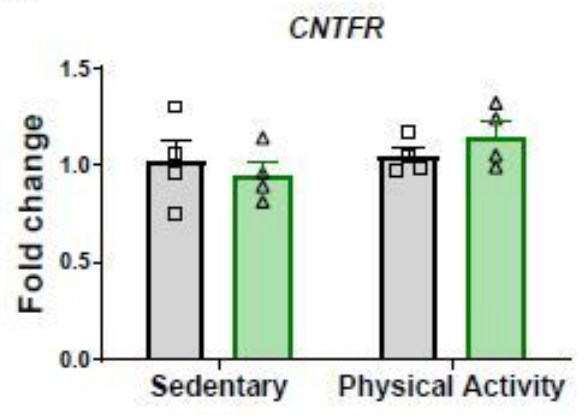

f)
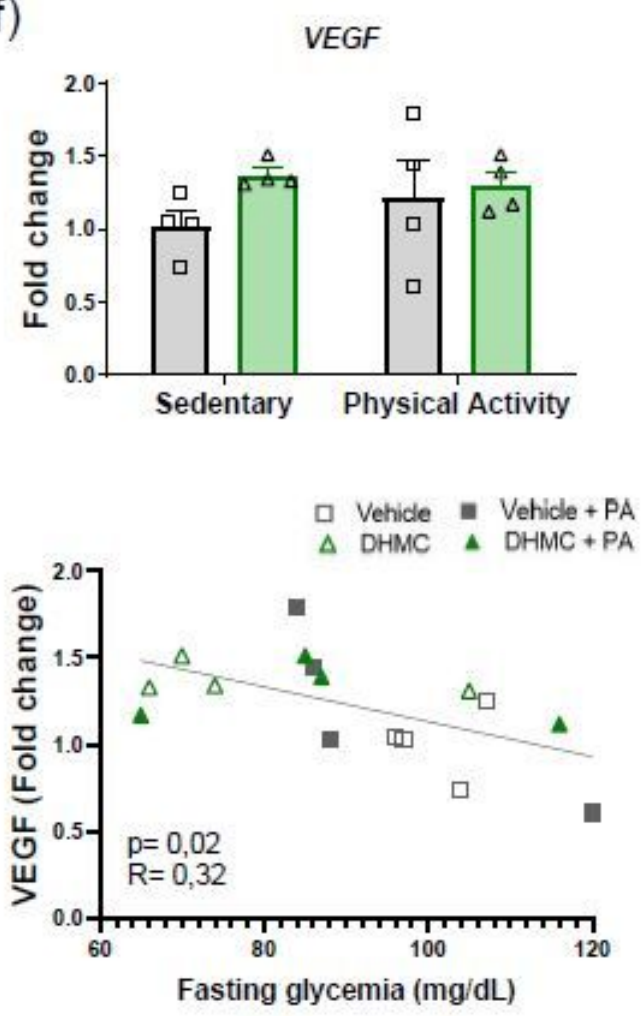

\section{Figure 3}

DHMC treatment increases hypothalamic VEGF gene expression. Two-way ANOVA (vehicle $x$ DHMC) $X$ neurotrophic marker (fold change) for evaluation of hypothalamic mRNA levels of DCX, NEUROD1, CNTF, CNTFR, BDNF and VEGF (A-F). Paired t-test (vehicle $x$ DHMC) considering animals exercised or not grouped, disregarding the physical activity variable. Representation of VEGF mRNA levels presented in $F$ in animals treated with vehicle and DHMC (G). Pearson's correlation coefficient analysis between VEGF 
mRNA expression and fasting glucose levels $(H) . N=4$ per group. Statistical significance defined when ${ }^{*} \mathrm{p}<0.05$. Data are presented as means $+\mathrm{SEM}$. Kolmogorov-Smirnov normality and variance equality Ftest. $\mathrm{DHMC}=7,8$-dihydroxy-4-methylcoumarin

\section{Supplementary Files}

This is a list of supplementary files associated with this preprint. Click to download.

- Figsl1.pdf 